\title{
Medicine Delivery Bot Using Time Series and Object Detection
}

doi : https://doi.org/10.32628/CSEIT217469

\author{
Kartikeya Bajpai"1 ${ }^{1}$ Prachi Jain ${ }^{2}$ \\ ${ }^{1}$ Computer Science Engineering, SRMIST, Lakhimpur-Kheri , Uttar Pradesh, India \\ ${ }^{2}$ Computer Science Engineering, SRMIST, Jaipur, Rajasthan, India \\ ${ }^{*}$ Corresponding Author: kartikeya0263@gmail.com
}

\begin{abstract}
Article Info

Volume 7, Issue 4

Page Number: 286-290

Publication Issue :

July-August-2021

Article History

Accepted : 18 July 2021

Published : 24 July 2021

Nowadays, delivery is mainly done by humans which includes a lot of manual work. The existing way is good but lacks faster deliveries. In the present context the deliveries are not possible $24^{*} 7$ by humans, especially in the case of medicines, customers often require immediate deliveries for maintaining their course of medication. Since, in many other fields AI has contributed to decreasing a lot of manual work and time. In this research paper, we have proposed the idea of a delivery bot which uses deep learning algorithms to detect traffic lights and classify the color of the traffic light. On the basis of which the lapse time will be calculated in between the two traffic lights and hence maps the route for delivery with the help of geocoding accordingly which helps in more secure and faster deliveries.
\end{abstract}

Keywords : Deep learning, YOLO, Delivery Bot, time series

\section{INTRODUCTION}

A medicine delivery bot is designed to make delivery of medicine without much human involvement. The idea of this is to increase the efficiency, accuracy and decrease time consumption during the process. There can be discrepancies in the manual deliveries such as delivery of wrong medicines, stealing of packages, damage to the package, fuel consumption by vehicles and more time consumption.

The bot proposed will overcome the listed issues as if we take the case of delivery of wrong medicines the bot will have a database of the medicines listed by the customer and during the process of dispatching it will scan the respective medicines to be delivered to the customer. Since the bot will be totally closed until the customer enters the OTP provided at the time of delivery and will also prevent the package from any damage. The bot will use solar cells as its source of energy and it will also have a battery backup of 3hrs which eliminates the fuel consumption.

\section{RELATED WORK}

A good amount of methodologies and research suggest some regulatory frameworks and last mile scheduling in Autonomous Delivery Bots. Research on regulatory framework enlists the analysis and further development of the regulatory framework of autonomous delivery robots for packages by highlighting legal implications. Since delivery robots can be understood as cyber-physical systems in the context of Industry 4.0, the research contributes to

Copyright: @ the author(s), publisher and licensee Technoscience Academy. This is an open-access article distributed under the terms of the Creative Commons Attribution Non-Commercial License, which permits unrestricted non-commercial use, distribution, and reproduction in any medium, provided the original work is properly cited 
the related regulatory framework of Industry 4.0 in international terms.[1]

The last mile scheduling concept is focused on excessive traffic in urban areas, hence launching autonomous delivery robots from trucks in which the autonomous robots move to their dedicated customers and, after delivery, autonomously return to some robot depot which requires development of scheduling procedures which determine the truck route along robot depots and drop-off points where robots are launched, such that the weighted number of late customer deliveries is minimized.[2]

\section{METHODOLOGY}

\section{A. GEOCODING}

Geocoding is a way of converting local addresses into geographic coordinates through which we can position the map. The API is accessed via the constructor object which is google.maps.Geocoder and then the request is made. In response to the above request a callback method is used which is displayed in the console via object. It contains all the fields which are necessary to position a location on the map. The data is stored in a database.[9]

\section{B. OBJECT DETECTION}

Detection of traffic lights and classifying them in red or green light Object detection algorithm is used in the model to analyse the data with the help of YOLO. You Only Look Once or YOLO is one of the popular algorithms in object detection used by researchers around the globe. According to the researchers at Facebook AI Research, the unified architecture of YOLO is extremely fast in manner. The base YOLO model processes images in real-time at 45 frames per second, while the smaller version of the network, Fast YOLO processes an astounding 155 frames per second while still achieving double the mAP of other real-time detectors. This algorithm outperforms the other detection methods, including DPM and R-CNN, when generalising from natural images to other domains like artwork.[7]

In step number one, the regions of interest are selected.Then those regions are classified using convolutional neural networks. This solution could be very slow because the prediction must be executed for every selected region. To analyse the performance of our trained model we used the concept of identification of true positive, true negative, false positive and false negative. Positive is when the object of interest is detected in the image i.e. bounding box is shown.Negative is when there is nothing detected in the image. True and false explains about the correctness of the desired object being detected in an image. True positive is when the object is detected correctly. True negative when there is no desired object in the image and hence it is not detected. False positive is when an object is detected wrongly as the desired object. False negative is when there is the desired object and it is not detected. This information helps to tell how well the dataset has been trained and what are the factors, or the parameters need to be modified if it doesn't show proper output.[8]

\section{TIME SERIES}

For incubating the lapse time concept for making delivery more time efficient we have added a Time Series segment to the model. Time series will help us predict the traffic congestion on the delivery path as well as the bot to utilise lapse time between traffic lights. An algorithm is to be designed which will use time series to analyse the lapse time between the traffic lights. Convolutional neural network (CNN) is a commonly applied DML algorithm in traffic engineering. Due to the excellent performance of $\mathrm{CNN}$ in image processing, while applying in traffic prediction, traffic flow data is converted into a 2-D matrix to process. There are five main parts of a CNN 
structure in transportation: the input layer, convolution layer, pool layer, full connection layer, and output layer. Both the convolution and pooling layer extracts important features. The depth of these two layers differs in different studies. Majority of the studies converted traffic flow data into an image of a 2-D matrix. The study folded the time-series to generate the input combining real-time and historical traffic data. To capture the correlation of a new time slot with the immediate past, they duplicated the congestion level of the last slot in the matrix and also applied five convolution-pooling layers as well as $\left(3^{*} 3\right)$ and $\left(2^{*} 2\right)$ sizes, respectively.[3][4]

\section{FABRICATION AND ACCESSORIES}

In fabrication of Delivery bot we will require following parts:

\section{MATERIAL}

For fabricating the outer parts of the bot we can use aluminium and Kevlar for the areas which require heat resistance and will be rugged. The cabins in which the medicines will be stored are to be made of Kevlar so as to keep medicines at the required temperature.

\section{MOTORS}

Delivery robots require mobility in order to perform their designed function. From remotely controlled to completely autonomous, and whether using wheels, tracks or legs, mobile robots require highperformance electric motors to enable movement with precision and efficiency.Motors and drives required for delivery bot are drives, brushless DC motors, Integrated motor drives,linear actuators, PMDC brush motors, Stepper motors, Mobility gearmotors, cable/harness solutions, HANSEN PMDC Brush motors.

Battery and Solar Panel-- battery backup, region sectors

\section{Capacity-- $10 \mathrm{~kg}$}

Speed-- 8kph

We will be using Intel RealSense LiDAR camera L515 which is a revolutionary solid state LiDAR depth camera which uses a proprietary MEMS mirror technology, enabling better laser power efficiency compared to other time of flight technologies. With less than $3.5 \mathrm{~W}$ power consumption for depth streaming, the Intel RealSense LiDAR camera L515 is the world's most power efficient high resolution LiDAR camera.

\section{GPS Controller}

A Promark 500 GPS (Magellan Co., Santa Clara, CA, USA) receiver is used to navigate the robot. The receiver had 75 channels and up to $20 \mathrm{~Hz}$ data output rate. Data such as the geographical coordinates and advance speed of the spot being measured were then sent to the serial port of the robot computer. The RS232 serial communication protocol was used for twoway communication between the GPS receiver and the robot computer using the serial data cable of the GPS receiver. The GPS receiver installation data were sent to set up the GPS receiver via the server software.[5]

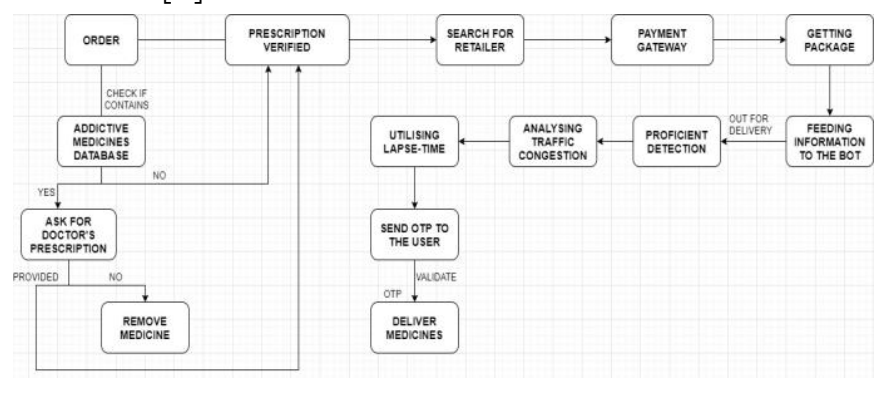

\section{DISCUSSIONS}

The proposed model uses Object detection i.e. Traffic light detection and Time series algorithms to ease the delivery process by using automated delivery bot. Object detection and applying time series in order to utilise the lapse time between the traffic lights enables a perfect door for faster and efficient delivery process hence making it more 
user friendly. In this model we have used some techniques which we would like to put forward and provide solutions to the posing loopholes.

For object detection i.e. Traffic light detection we have used YOLO in the proposed model as it is incredibly fast and processes 45 frames per second which increases the efficiency of the whole process which ensures a better analysis of the data than RCNN algorithms.

When user places a order for several medicines through app there might be some medicines which have addictive properties in long term use which can only be taken by the doctor's prescription, for the same, we will have a separate database of such medicines which when entered in order list by user it will ask for a digital copy of the prescription by the concerned doctor or else that medicine won't be delivered by the bot.

For time efficient delivery processes we have used the time series concept to work on traffic congestion prediction and lapse time between two traffic lights so as to provide a clear path for the bot to travel faster.

GPS isn't accurate enough for the bot, they'll have to detect traffic lights and recognise pedestrian crossings. To avoid these obstacles the bot will have sufficient data. If this doesn't work, the bot has a camera and microphone to assist them in engaging with humans and overcome these obstacles.[6]

\section{RESULT}

We have proposed a model keeping most of the perspectives in mind of users as well as of service providers.
Advantages of the proposed model:

1- Robot's increasing success in the industry can be due to their low labor cost and high performance. Furthermore, it is easy and flexible to use. Customers are going to be able to make a choice from a variety of precise delivery slots which means that delivery would be done according to the time that suits them.

2- During delivery customers can track the location of the bot and on arrival the cargo will be unlocked using an OTP which will be sent to the customers mobile number hence ensuring security which is rid of any human discrepancies.

3- The bot will have solar panels which makes it ecofriendly as it will not use any batteries.

4- Another advantage of using the bot is it's attractiveness to the customers.

5- Based on this methodology, the bot has high accuracy and low impact. It is obvious that there will be further improvements regarding the designs and storage capacity which can be incubated to make it better in near future.

Disadvantages of the proposed model:

1- The bot has limited storage capacity.

2- Databases and passwords need to be updated for each package during every single delivery.

\section{CONCLUSION}

We thus proposed an improved model of medicine delivery bot than the traditional bots and enabling easier and faster delivery of medicines even in remote areas. The delivery will be well ensured in terms of efficiency because of usage of prediction models which will provide a clear path to the bot. It has security measures incubated by providing OTP to the customer as a password to get the medicines. It is a user friendly and inexpensive approach towards the delivery process. Our proposed model,therefore, eliminates most of the drawbacks of the manual delivery system especially in case of urgency. 


\section{REFERENCES}

[1]. NilsBoysen, StefanSchwerdfeger, FelixWeidinger, "Scheduling last-mile deliveries with truck-based autonomous robots", European Journal of Operational Research Volume 271, Issue 3, 16 December 2018, Pages 1085-1099

[2]. Thomas Hoffmann, Gunnar Prause, "On the Regulatory Framework for Last-Mile Delivery Robots", Department of Business Administration, Tallinn University of Technology, Machines, 10.3390/machines6030033, 2018 , Vol 6 (3), pp. 33

[3]. Mahmuda Akhtar, Sara Moridpour, "A Review of Traffic Congestion Prediction Using Artificial Intelligence", Journal of Advanced Transportation, vol. 2021, Article ID 8878011, 18

pages,

2021.

https://doi.org/10.1155/2021/8878011

[4]. Manish R. Joshi, Theyazan Hassn Hadi, “A Review of Network Traffic Analysis and Prediction Techniques", School of Computer Sciences, North Maharashtra University, Jalgaon (M.S), India

[5]. Ilker Ünal, Mehmet Topakci, "Design of a Remote-Controlled and GPS-Guided Autonomous Robot for Precision Farming", Volume: 12 issue: 12

[6]. Evan Ackerman, "Startup Developing Autonomous Delivery Robots That Travel on Sidewalks", IEEE Spectrum

[7]. Wael Omar, Impyeong Lee, Gyuseok Lee, Kang Min Park, "DETECTION AND LOCALIZATION OF TRAFFIC LIGHTS USING YOLOV3 AND STEREO VISION", The International Archives of the Photogrammetry, Remote Sensing and Spatial Information Sciences, Volume XLIII-B2-2020, 2020
[8]. Akansha Bathija, Prof. Grishma Sharma, "Visual Object Detection and Tracking using YOLO and SORT", International Journal of Engineering Research \& Technology (IJERT), Vol. 8 Issue 11

[9]. Vishal Srivastava, Priyam Tejaswin, Lucky Dhakad, Mohit Kumar, Amar Dani, “A Geocoding Framework Powered by Delivery Data", Proceedings of the 28th International Conference on Advances in Geographic Information Systems, Pages 568-577

\section{Cite this article as :}

Kartikeya Bajpai, Prachi Jain, "Medicine Delivery Bot Using Time Series and Object Detection", International Journal of Scientific Research in Computer Science, Engineering and Information Technology (IJSRCSEIT), ISSN : 2456-3307, Volume 7 Issue 4, pp. 286-290, July-August 2021. Available at doi : https://doi.org/10.32628/CSEIT217469 Journal URL : https://ijsrcseit.com/CSEIT217469 